\title{
Superparamagnetism in FeCo nanoparticles
}

\author{
P. P. Pradyumnan \\ Department of Physics, University of Calicut, Kerala 673 635, India \\ drpradyumnan@gmail.com
}

PACS 75.75.+a, 81.16 Be

DOI 10.17586/2220-8054-2016-7-4-675-677

\begin{abstract}
Superparamagnetism is an important physical property of a certain kinds of nanoparticles and these particles have attracted interest because of their applications in the technological world and medical fields. In this work, the author reports the synthesis of iron cobalt $\left(\mathrm{Fe}_{60} \mathrm{Co}_{40}\right)$ and Manganese (Mn) incorporated FeCo compound nanoparticles by a simple inert atmosphere reductive decomposition method. The synthesized nanoparticles were characterized by transmission electron microscopy (TEM), X-ray diffraction technique (XRD), selected area electron diffraction (SAED), energy dispersive X-ray analysis (EDX) and Fourier transform infrared (FTIR) spectroscopic method. The magnetic properties of the particles have been studied with a magnetometer (SQUID).
\end{abstract}

Keywords: Nanoparticle, soft magnetic, $\mathrm{Fe}_{1-x} \mathrm{Co}_{x}$ compound, reductive decomposition, TEM, SQUID

Received: 5 February 2016

Revised: 24 April 2016

\section{Introduction}

Nanoparticles have now turned from theoretical interest to application in the technological world. In these years nanomagnetic particles have drawn special attention because of their recording ability and use as perpendicular magnetic memory and biomedical applications such as hyperthermia, magnetic resonance contrast enhancement and drug delivery etc. [1]. The nanoparticles, which show high magnetic moment are a better candidate, as far as the applications are concerned. The FeCo nano particle has such ability, but the main task is its preparation and stability. Many investigators have prepared nanoparticles of $\mathrm{FeCo}$ compounds using different techniques.

They have used chemical and physical methods for the growth of nanoparticles. Most of the physical methods require substrates [2-6]. For the fabrication of perpendicular recording media, substrate is applicable, but for the biological application individual nanoparticles are more reliable.

This paper reports the preparation of multifunctional nanoparticles of $\mathrm{Fe}_{1-x} \mathrm{Co}_{x}$ and Mn-incorporated compounds by a reductive decomposition-based reflux method using suitable stabilizers and surfactants. We have prepared three compositional compounds containing iron and cobalt as well iron, cobalt and manganese. All these synthesized materials were characterized by X-ray diffraction (XRD), selective area electron diffraction (SAED), Fourier transform infrared spectroscopy (FTIR), energy dispersive X-ray (EDX) and transmission electron microscopy (TEM). The magnetic properties of the materials studied using SQUID magnetometer.

\section{Synthesis}

The $\mathrm{Fe}_{1-x} \mathrm{Co}_{x}$ compounds, $\mathrm{Mn}$ incorporated nanoparticles were prepared using a modified method reported by Sun et al. [2]. Here, we used $\mathrm{Fe}(\mathrm{acac})_{3}$ and $\mathrm{Co}(\mathrm{acac})_{2}, \mathrm{Mn}(\mathrm{acac})_{2}$ as precursors. Reductive decomposition of these was achieved by using benzyl ether as solvent medium, 1-octadecene as reducing agent and oleic acid as surfactant and oleylamine as stabilizer. The growth temperature was fixed at $240{ }^{\circ} \mathrm{C}$. To avoid oxidation during nanoparticle growth, the reaction chamber was blanketed with argon. Care has been taken for the collection of the nanoparticles and washing of the nanoparticles to avoid the surfactants and stabilizers. But it was found to be very difficult to completely rinse the surfactants and stabilizers from the nanoparticles.

The shape of the nanoparticles depended on the growth temperature, whereas it had nothing to do with the composition and size of the particles. But it is very difficult to control the size of all the particles in this case. In our studies, we have fixed the above temperature for obtaining the maximum product. In all the compositions, the particle size found was to be less than $10 \mathrm{~nm}$. 


\section{Experiments}

\subsection{X-ray diffraction (XRD)}

$\mathrm{XRD}$ is the most primary technique to analyze the crystalline nature of the material. A Rigaku X-ray diffractometer has been utilized for the studies. All the three compounds shows the crystal structure is body centered cubic (bcc). Since the particle size is too small, the spectral broadening is evident. The peaks at (110) and (200) peaks are visible. TEM-electron diffraction studies were also utilized to confirm the crystalline nature of the compounds.

\subsection{Infrared Spectroscopy}

During the preparation of the compounds, organic chemicals like oleic acid and oleylamine were utilized. Even though proper washing has been done for the particles, there is a finite possibility that these molecules can be found on the surface of the nanoparticles. The IR spectrum of all the particles shows, the absorption bands. It is very difficult to wash out all the surfactants, and there is a strong attachment between the compound particles and these molecules. Fig. 1 shows the IR spectrum for one sample of the compounds.

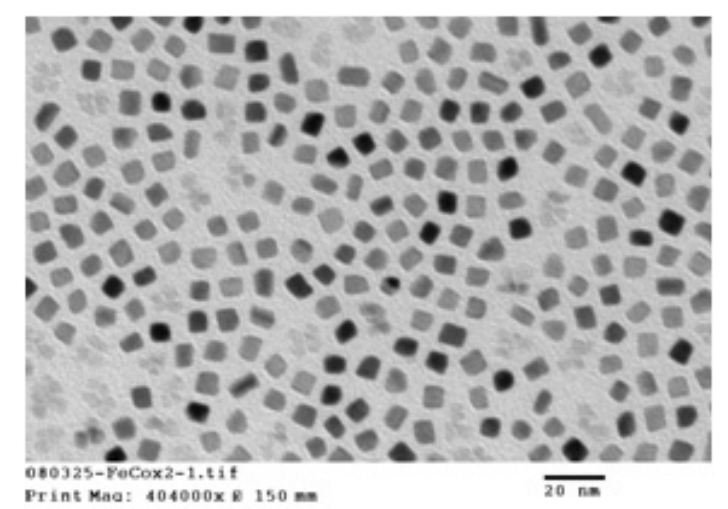

FIG. 1. TEM image of FeCo nano particles

For oleic acid, whose formula is $\left(\mathrm{CH}_{3}\left(\mathrm{CH}_{2}\right)_{7} \mathrm{CH}=\mathrm{CH}\left(\mathrm{CH}_{2}\right)_{7} \mathrm{COOH}\right)$, the following pattern was observed: the vibration is assigned at $\mathrm{C}-\mathrm{C}$ (either $\mathrm{COOH}-$ or $\mathrm{CH}_{3}$-sided) stretching. The difference observed is probably due to $\mathrm{C}=\mathrm{C}$ bonding. In the spectrum of the broad, strong band at $1161 \mathrm{~cm}^{-1}$ flanked by weaker bands at 1238 and $1118 \mathrm{~cm}^{-1}$.

\subsection{Energy Dispersive X-ray Analysis (EDX)}

The percentages of the $\mathrm{Fe}$ and $\mathrm{Co}$ present in all the prepared compound nanoparticles were measured using EDX technique. During synthesis we have approximated the ratio by varying the molar ratio of the precursors. Table 1, gives the ration of the final compositions of the $\mathrm{Fe}_{1-x} \mathrm{Co}_{x}$ compounds. From these results, we observed that the $\mathrm{Fe}$ ion is dominant in the compound formation and the single phases will be observed during these ratios only.

TABLE 1. Ration of the expected and actual compositions for $\mathrm{Fe}_{1-x} \mathrm{Co}_{x}$ compounds

\begin{tabular}{|c|c|c|c|c|}
\hline \multirow{2}{*}{$\begin{array}{c}\text { No of compound } \\
\text { combinations }\end{array}$} & \multicolumn{2}{|c|}{$\begin{array}{c}\text { Molar ratio of the metal } \\
\text { precursors used }\end{array}$} & \multicolumn{2}{c|}{$\begin{array}{c}\text { Molar ratio of the metal actually } \\
\text { present in the compounds as per EDX }\end{array}$} \\
\cline { 2 - 5 } & $\mathrm{Fe}$ & $\mathrm{Co}$ & $\mathrm{Fe}$ & $\mathrm{Co}$ \\
\hline 1 & 0.60 & 0.40 & 0.65 & 0.35 \\
\hline 2 & 0.50 & 0.50 & 0.60 & 0.40 \\
\hline 3 & 0.40 & 0.60 & 0.55 & 0.45 \\
\hline
\end{tabular}




\subsection{Transmission Electron Microscopic Studies (TEM)}

The TEM images of the prepared nano particles are shown in Fig. 1. The $\mathrm{Fe}_{1-x} \mathrm{Co}_{x}$ compounds $(x=0.35$, $0.40,0.45)$.

The coercivity was found to be 0 . Fig. 2 shows the room temperature hysteresis loops of the $\mathrm{Fe}_{60} \mathrm{Co}_{40}$ compound nanoparticles. We took same weight $(0.1 \mathrm{mg})$ of both compound particles for SQUID studies It is found that due to the incorporation of $\mathrm{Mn}$, the magnetic behavior of the materials were drastically changed and the magnetic moment was reduced, due to the incorporation of Mn ions. Fig. 2 shows the M/Ms Vs H graph of synthesized materials.
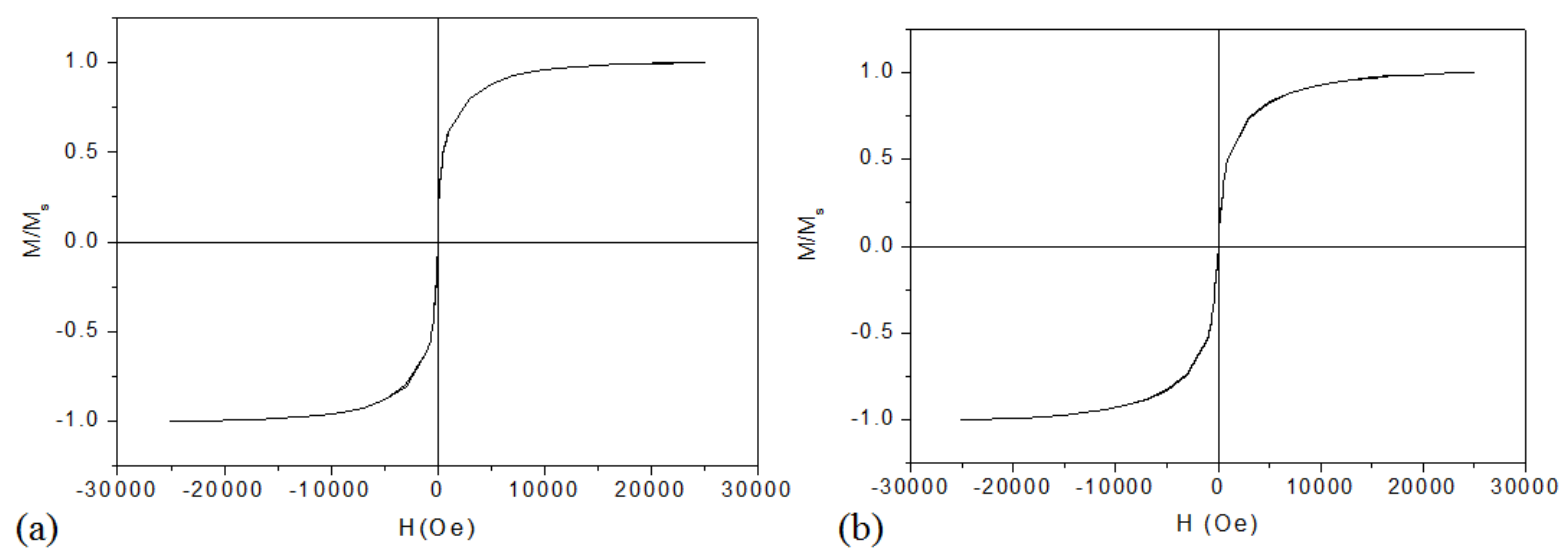

FIG. 2. Hysteresis loop of (a) $\mathrm{Fe}_{60} \mathrm{Co}_{40}$ and (b) $\mathrm{Fe}_{65} \mathrm{Co}_{30} \mathrm{Mn}_{5}$ compound nanoparticle (size: 4 - $5 \mathrm{~nm}$ )

\section{Conclusion}

Well defined nanoparticles of FeCo and Mn incorporated FeCo compound were synthesized by a reductive decomposition-based reflux method. Uniformly sized nanoparticles of size $4-5 \mathrm{~nm}$ have been well characterized. The magnetic properties were attenuated due to the incorporation of $\mathrm{Mn}$ ion. Both the compound particles showed super paramagnetic behavior at room temperature.

\section{Acknowledgements}

Author grateful to SERB, Govt. of India for major research project funding SB/EMEQ-002/2013 and DSTFIST, Department of Physics, University of Calicut for experimental facilities.

\section{References}

[1] Reiss G., Hutten A. Magnetic nanoparticles-applications beyond data storage. Nature materials, 2005,4 (10), P. 725-726.

[2] Don Keun Lee, Young Hwan Kim, et al. Preparation of monodisperse Co and Fe nanoparticle using precursor of $\mathrm{M}^{2+}$-oleate $2(\mathrm{M}=\mathrm{Co}$, Fe). Curr. Appl. Phys., 2006, 6, P. 786-790

[3] Soo Ja Shin, Young Hwan Kim, et al. Preparation of magnetic FeCo nanoparticles by coprecipitation route. Curr. Appl. Phys., 2007, 7, P. 404-408.

[4] Yun Hao Xu, Jianmin Bai, Jian-Ping Wang. High-magnetic-moment multifunctional nanoparticles for nanomedicine applications. J. Magn. Magn. Mater, 2007, 311, P. 131-134.

[5] Wen J.Z., Goldsmith F.C., et al. Detailed Kinetic Modeling of Iron Nanoparticle Synthesis from the Decomposition of Fe(CO) 5 . J. Phys. Chem. C, 2007, 111, P. 5677-5688.

[6] Shousheng Sun, Murrey C.B., et al. Monodisperse FePt Nanoparticles and Ferromagnetic FePt Nanocrystal Superlattices. Science, 2000, 289, P. 1989-1992. 